\title{
Combinations of Case-Based Reasoning with Other Intelligent Methods
}

\author{
Jim Prentzas ${ }^{1}$ and Ioannis Hatzilygeroudis ${ }^{2}$
}

\begin{abstract}
Case-based reasoning is a popular approach used in intelligent systems. Whenever a new case has to be dealt with, the most similar cases are retrieved from the case base and their encompassed knowledge is exploited in the current situation. Combinations of case-based reasoning with other intelligent methods have been explored deriving effective knowledge representation schemes. Although some types of combinations have been mostly explored, other types have not been thoroughly investigated. In this paper, we briefly outline popular case-based reasoning combinations. More specifically, we focus on combinations of case-based reasoning with rulebased reasoning, soft computing and ontologies. We illustrate basic types of such combinations and discuss future directions.
\end{abstract}

\section{INTRODUCTION}

Case-based representations store a large set of previous cases with their solutions in the case base using them whenever a similar new case has to be dealt with [19], [22]. Whenever, a new input case comes in, a case-based system performs inference in four phases known as the case-based reasoning (CBR) cycle [1]: (i) retrieve, (ii) reuse, (iii) revise and (iv) retain. The retrieval phase retrieves from the case base the most relevant stored case(s) to the new case. Indexing schemes and similarity metrics are used for this purpose. In the reuse phase, a solution for the new case is created based on the retrieved most relevant case(s). The revise phase validates the correctness of the proposed solution, perhaps with the intervention of the user. Finally, the retain phase decides whether the knowledge learned from the solution of the new case is important enough to be incorporated into the system.

CBR can be effectively combined with other intelligent methods [25], [31]. Two main trends for CBR combinations can be discerned. The first trend involves embedded approaches in which the primary intelligent method (usually CBR) embeds one or more other intelligent methods to assist its internal online and offline tasks. The second combination trend involves approaches in which the problem solving process can be decomposed into tasks for which different representation formalisms are required or available. In such situations, a CBR system as a whole (with its possible internal modules) is integrated 'externally' with other intelligent systems to create an improved overall system.

Popular CBR combinations involve combinations with rulebased reasoning (RBR), model-based reasoning (MBR) and soft computing methods. CBR has also been combined with other intelligent methods (e.g. ontologies). In certain CBR combinations both combination trends have been followed. In other combinations one of the two trends is mostly explored.

In this paper, we briefly discuss aspects involving CBR combinations. We focus on intelligent methods with which CBR is usually combined. Our purpose is not to present an extensive survey of developed CBR combinations but to present their key aspects.

\section{COMBINATIONS OF CBR}

Combinations of CBR with other intelligent methods have been explored for more effective knowledge representation and problem solving. CBR can be combined with various intelligent methods. However, CBR is usually combined with RBR, MBR and soft computing methods.

To categorize CBR combinations one could use Medsker's general categorization scheme for integrated intelligent systems [26]. Medsker distinguishes five main combination models: standalone, transformational, loose coupling, tight coupling and fully integrated models. Distinction between those models is based on the degree of coupling between the integrated components. Underlying categories for some of these models are also defined. Main types of underlying categories for loose and tight coupling models involve pre-processing, postprocessing and co-processing models as well as embedded processing (for tight coupling models only). Not all of these combination models and/or their underlying categories have been thoroughly explored in the case of CBR combinations. The types of combination models that have been applied to CBR combinations depend on the nature of the other intelligent methods combined with CBR. Some combination models are difficult to apply in certain CBR combinations. For instance, it is difficult to apply the fully integrated model in combinations of RBR with CBR. Obviously, the standalone model can be applied to combinations of CBR with any other method.

Generally speaking, coupling models are the most usual CBR combination models. More specifically, embedded coupling approaches constitute perhaps the most popular trend.

\footnotetext{
${ }^{1}$ Technological Educational Institute of Lamia, Department of Informatics and Computer Technology, 35100 Lamia, Greece, email: dprentzas@teilam.gr.

${ }^{2}$ University of Patras, Dept of Computer Engineering \& Informatics, 26500 Patras, Greece, email: ihatz@ceid.upatras.gr.
} 
Most of the combinations following this trend use other intelligent methods to assist various CBR tasks. CBR is a generic methodology for building knowledge-based systems and its internal reasoning tasks can be implemented using a number of techniques as long as the guiding CBR principles are followed [36]. The reverse approach that is, embedding casebased modules into intelligent systems employing other representations to assist in their internal tasks does not seem to be popular with the exception of combinations with genetic algorithms. In combinations of CBR with RBR and MBR, various coupling approaches have also been investigated besides embedded approaches [31]. In coupling combinations of CBR with soft computing methods, embedded approaches seem to be the most thoroughly investigated.

In the following, we discuss main issues involving combinations of CBR with RBR, fuzzy logic, neural networks, genetic algorithms and ontologies.

\subsection{Combinations of CBR with RBR}

Various types of coupling models involving combinations of CBR and RBR have been investigated i.e., sequential processing, co-processing and embedded processing [31].

In sequential processing, information (produced by reasoning) necessarily passes sequentially through some or all of the combined modules to produce the final result [33], [11].

In co-processing approaches, the combined modules closely interact in producing the final result. Such systems can be discerned into two types: cooperation-oriented, which give emphasis on cooperation, and reconciliation-oriented, which give emphasis on reconciliation. In the former type, the combined components cooperate with each other (usually by interleaving their reasoning steps) [27], [32]. In the latter, each component produces its own conclusion, possibly differing from the conclusion of the other component, and thus a reconciliation process is necessary [14].

In embedded processing, CBR systems employ one or more RBR modules to perform tasks of their CBR cycle (e.g. retrieval and adaptation). Such approaches are quite common in CBR especially for adaptation. RBR systems embedding CBR modules do not seem to exist.

\subsection{Combinations of CBR with Fuzzy Logic}

CBR can be combined with fuzzy logic in fruitful ways in order to handle imprecision. A usual approach is the incorporation of fuzzy logic into a CBR system in order to improve CBR aspects [4], [29], [35], [9]. Such combinations have been vastly explored as imprecision and uncertainty are inherent in various CBR tasks. Fuzzy terms may be used in case representation enabling a flexible encoding of case features that encompasses imprecise and uncertain information. Fuzzy logic may be also proved very useful in indexing and retrieval. Fuzzy indexing enables multiple indexing of a case on a single feature with different degrees of membership [35]. Fuzzy similarity assessment and matching methods can produce more accurate results. Fuzzy clustering and classification methods can also be applied in case retrieval. In addition, fuzzy adaptation rules can be employed in case adaptation.

The works concerning combination of RBR with CBR [31] could potentially be improved with use of fuzzy rules. Investigation of coupling approaches in combinations of CBR with fuzzy systems besides embedded ones could be fruitful.

\subsection{Combinations of CBR with Neural Networks}

Neural networks are usually employed by CBR to perform tasks such as indexing, retrieval and adaptation. In this way, appealing characteristics of neural networks such as parallelism, robustness, adaptability, generalization and ability to cope with incomplete input data are exploited [10], [35]. Due to the fact that different types of neural networks have been developed (e.g. back propagation neural networks, radial basis function networks, Self-Organizing Map networks, ART network), different types of neural capabilities for classification and clustering can be exploited. Certain CBR approaches have employed different types of neural networks for the various internal CBR tasks (e.g. [12], [34]). Knowledge extracted from neural networks could also be exploited by CBR [10], [35]. An interesting direction could involve non-embedded coupling approaches combining CBR with neural networks.

\subsection{Combinations of CBR with Genetic Algorithms}

Usual combinations of CBR with genetic algorithms (GAs) involve use of GAs to optimize (one or more) aspects of a CBR system. On the other hand, CBR can be exploited to enhance GAs. Other types of combinations of CBR with GAs can be also implemented.

GAs can be used within CBR to enhance indexing and retrieval. GAs have been used to assign case feature weights enhancing similarity assessment [39], [8], to perform feature selection [18] and generally to select relevant indices for evolving environments. GAs have also been used to retrieve multiple similar cases [38]. If $\mathrm{k}$ nearest neighbor retrieval is applied, genetic algorithms can be used to find the optimal $\mathrm{k}$ parameter in order to improve the retrieval accuracy [2]. Furthermore, GAs can be used to perform instance selection i.e., finding the representative cases in a case base and determining a reduced subset of a case base. In this way, time performance is improved by reducing search space and accuracy can be improved through elimination of noisy and useless cases [2].

Additionally, GAs have been used to enhance case adaptation [16], [17]. Genetic algorithms can also optimize case representation, e.g. by performing case feature discretization [18] and removing irrelevant features. Such optimizations improve accuracy, search time and storage requirements. It is also quite usual to simultaneously optimize more than one CBR aspect with GAs (e.g. [2], [18]).

On the other hand CBR can be employed to enhance GAs. CBR can be applied to GAs by creating cases to track the history of a search. This case base can contribute in the understanding of how a solution was reached, why a solution works, and what the search space looks like. It could thus be 
used to design highly tailored search strategies for future use [23]. Such an approach could therefore be used to explain the results of the genetic algorithm and for knowledge extraction. Moreover, similar stored cases can be also incorporated into a genetic algorithm to reduce convergence time and improve solution accuracy. GAs randomly initialize their starting population. Instead, relevant stored cases can be used as part of the initial population (solution) of GAs. Additionally, relevant stored cases can be periodically injected into the pool of chromosomes while the genetic algorithm runs [24], [7]. In certain approaches, CBR is exploited by GAs for both knowledge extraction and case injection [30].

\subsection{Combinations of CBR with Ontologies}

Ontologies facilitate knowledge sharing and reuse. They can provide an explicit conceptualization describing data semantics and a shared and common understanding of the domain knowledge that can be communicated among agents and application systems [6]. Ontologies play a crucial role in enabling the processing and sharing of knowledge between programs on the Web [21]. Intelligent Decision Support Systems in the semantic Web framework should be able to handle, integrate with and reason from distributed data and information on the Web [3].

Therefore ontologies can be combined with CBR in various ways. Ontologies can be used by a CBR system to represent the input problem [20], to enhance similarity assessment [13], case representation, case abstraction and case adaptation [3]. Ontologies may perform all such CBR tasks [37].

\subsection{Combinations of CBR with Multiple Intelligent Methods}

The previous sections focused on combinations of CBR with one other individual intelligent method. However, intelligent systems have been developed that combine CBR with multiple other intelligent methods. Such multi-integrated paradigms usually follow a coupling model.

Obviously, a CBR system may employ multiple intelligent methods (e.g. rules and various soft computing methods) to perform its internal tasks [36]. Typical examples of approaches employing multiple soft computing methods within the CBR cycle are presented in [12] and [34]. In [12] all of the four phases of the CBR cycle employ soft computing methods. Employed soft computing methods are a self-organizing neural network for retrieval, a radial basis neural network for reuse, fuzzy systems for revise and all soft computing methods for retain. In [34] fuzzy logic, supervised and unsupervised neural networks and a genetic algorithm are employed for case representation, indexing, retrieval and adaptation.

More interesting approaches concern multi-integrated systems not following the embedded approach. Typical such multi-integrated approaches involve combinations of CBR, RBR and MBR (e.g. [28]). Such approaches seem to be quite effective, because combinations of CBR with RBR and MBR individually have been thoroughly investigated. Quite often such systems have been implemented to deal with deficiencies of earlier systems combining CBR with only one of the other two intelligent methods (e.g. RBR or MBR alone). Multiintegrated CBR approaches, besides those involving RBR/MBR, could be developed. For instance, ontologies could constitute an interesting candidate method that could be combined with CBR and another intelligent method in order to facilitate knowledge sharing and reuse among the integrated system components themselves [5] and among integrated systems. Such a combination could be useful in Web-based systems that need to share knowledge. Fruitful such approaches could involve combinations of CBR, ontologies and RBR/MBR. For instance in [6] an approach combining CBR, $\mathrm{RBR}$ and an ontology is presented.

Multi-integrated paradigms could also be considered systems combining CBR with certain types of neuro-symbolic or neurofuzzy approaches in which the neuro-symbolic (neuro-fuzzy) module fully integrates the neural and symbolic (fuzzy) approach. Such modules could be used within CBR instead of plain neural or fuzzy components. Non-embedded coupling approaches can be applied as well. For instance, in [15] a neuro-symbolic method is combined with CBR according to the reconciliation coupling approach.

\section{CONCLUSIONS}

In this paper, we discuss key aspects involving combinations of CBR with other intelligent methods. Such combinations are becoming increasingly popular due to the fact that in many application domains a vast amount of case data is available. Such combined approaches have managed to solve problems in application domains where a case-based module needs the assistance and/or completion of other intelligent modules in order to produce effective results. This trend is very likely to carry on in the following years.

Future directions in combinations of CBR with other intelligent methods could involve a number of aspects. Main such aspects involve: (a) combinations of CBR with soft computing methods, (b) combinations of CBR with fuzzy rules, (c) combinations of CBR with ontologies and (d) combinations of CBR with neuro-symbolic and neuro-fuzzy approaches.

Combinations of CBR with soft computing methods not following an embedded coupling approach could be an interesting future research direction. At present there seems to be a lack of great interest in pursuing this direction since the main interest has been focused on employing soft computing methods within CBR. A non-embedded direction in the combinations of CBR with soft computing could be pursued as thoroughly as in the case of combinations of CBR with RBR/MBR. A further step towards this direction could involve non-embedded approaches combining CBR with multiple soft computing methods or combinations of CBR, soft computing and other intelligent methods (e.g. RBR, MBR or ontologies).

Combinations of CBR with fuzzy rule-based systems could be based on work combining CBR with RBR that is, investigation of various coupling approaches.

The increasing interest in Web-based intelligent systems and future advances in the Semantic Web is likely to provide an impetus to approaches combining CBR with ontologies. This 
trend is likely to involve multi-integrated approaches combining CBR, ontologies and other intelligent methods.

Finally, a direction that may be useful to pursue involves non-embedded coupling approaches combining CBR with neuro-symbolic and neuro-fuzzy modules. Few such approaches have been developed.

\section{REFERENCES}

[1] A. Aamodt, E. Plaza, 'Case-Based Reasoning: Foundational Issues, Methodological Variations, and System Approaches’, AI Communications, 7, 39-59, (2004).

[2] H. Ahn, K. Kim, 'Global optimization of case-based reasoning for breast cytology diagnosis’, Expert Systems with Applications (to appear).

[3] I. Bischindaritz, 'Memoire: A Framework for Semantic Interoperability of Case-Based Reasoning Systems in Biology and Medicine', Artificial Intelligence in Medicine, 36, 177-192, (2006).

[4] P.P. Bonissone, R. Lopez de Mantaras, 'Fuzzy Case-Based Reasoning Systems', Handbook on Fuzzy Computing, vol. F 4.3, Oxford University Press, 1998.

[5] L. Castillo, E. Armengol, E. Onaindia, L. Sebastia, J. GonzalezBoticario, A. Rodriguez, S. Fernandez, J.D. Arias, D. Borrajo, 'SAMAP: An User Oriented Adaptive System for Planning Tourist Visits', Expert Systems with Applications, 34, 1318-1332, (2008).

[6] L. Ceccaroni, U. Cortes, M. Sanchez-Marre, 'OntoWEDSS : Augmenting Environmental Decision-Support Systems with Ontologies', Environmental Modelling \& Software, 19, 785-797, (2004).

[7] P.-C. Chang, J.-C. Hsieh, C.-H. Liu, 'A Case-Injected Genetic Algorithm for Single Machine Scheduling Problems with Release Time', International Journal on Production Economics, 103, 551564, (2006).

[8] P.-C. Chang, C.-Y. Lai, K. R. Lai, 'A Hybrid System by Evolving Case-Based Reasoning with Genetic Algorithm in Wholesaler's Returning Book Forecasting', Decision Support Systems, 42, 17151729, (2006)

[9] W. Cheetam, S.C.K. Shiu, R.O. Weber, 'Soft Case-Based Reasoning', Knowledge Engineering Review, 20, 267-269, (2006).

[10] D. Chen, P. Burrell, 'Case-Based Reasoning System and Artificial Neural Networks: A Review’, Neural Computing \& Applications, 10, 264-276, (2001).

[11] R.-J. Dzeng, H.-Y. Lee, 'Critiquing Contractors' Scheduling by Integrating Rule-Based and Case-Based Reasoning', Automation in Construction, 13, 665-678, (2004).

[12] F. Fdez-Riverola, J.M. Corchado, 'FSfRT: Forecasting System for Red Tides’, Applied Intelligence, 21, 251-264, (2004).

[13] P. Gervas, B. Diaz-Agudo, F. Peinado, R. Hervas, 'Story Plot Generation Based on CBR', Knowledge-Based Systems, 18, 235-242, (2005).

[14] A.R. Golding, P.S. Rosenbloom, 'Improving Accuracy by Combining Rule-Based and Case-Based Reasoning', Artificial Intelligence, 87, 215-254, (1996).

[15] I. Hatzilygeroudis, J. Prentzas, 'Integrating (Rules, Neural Networks) and Cases for Knowledge Representation and Reasoning in Expert Systems', Expert Systems with Applications, 27, 63-75, (2004).

[16] B. W. Huang, M. L. Shih, N.-H. Chiu, W. Y. Hu, C. Chiu, 'Price information evaluation and prediction for broiler using adapted casebased reasoning approach', Expert Systems with Applications (to appear).

[17] Y.-K. Juan, S.-G. Shih, Y.-H. Perng, 'Decision Support for Housing Customization: A hybrid approach using case-based reasoning and genetic algorithm', Expert Systems with Applications, 31, 83-93, (2006).

[18] K.-J. Kim, 'Toward Global Optimization of Case-Based Reasoning Systems for Financial Forecasting', Applied Intelligence, 21, 239-249, (2004).

[19] J.L. Kolodner, Case-Based Reasoning, Morgan Kaufmann, 1993.

[20] O. Byung Kwon, 'Meta Web Service: Building Web-based Open Decision Support System based on Web Services', Expert Systems with Applications, 24, 375-389, (2003).

[21] O. Kwon, M. Kim, 'MyMessage: Case-Based Reasoning and Multicriteria Decision Making Techniques for Intelligent ContextAware Message Filtering', Expert Systems with Applications, 27, 467480, (2004).

[22] D.B. Leake (Ed.), Case-Based Reasoning: Experiences, Lessons, and Future Directions, AAAI Press, 1996.

[23] S.J. Louis, G. McGraw, R.O. Wyckoff, 'Case-based reasoning assisted explanation of genetic algorithm results', Journal of Experimental \& Theoretical Artificial Intelligence, 5, 21-37, (1993).

[24] S. J. Louis, C. Miles, 'Playing to Learn: Case-Injected Genetic Algorithms for Learning to Play Computer Games', IEEE Transactions on Evolutionary Computation, 9, 669-681, (2005).

[25] C. Marling, M. Sqalli, E. Rissland, H. Munoz-Avila, D. Aha, 'CaseBased Reasoning Integrations’, AI Magazine, 23, 69-86, (2002).

[26] L.R. Medsker, Hybrid Intelligent Systems, Kluwer Academic Publishers, Second Printing, 1998.

[27] S. Montani, R. Bellazzi, 'Supporting Decisions in Medical Applications: the Knowledge Management Perspective', International Journal of Medical Informatics, 68, 79-90, (2002).

[28] S. Montani, P. Magni, R. Bellazzi, C. Larizza, A.V. Roudsari, E.R. Carson, 'Integrating model-based decision support in a multi-modal reasoning system for managing type 1 diabetic patients', Artificial Intelligence in Medicine, 29, 131-151, (2003).

[29] S. K. Pal, S.C.K. Shiu, Foundations of Soft Case-Based Reasoning, John Wiley, 2004.

[30] E.I. Perez, C.A. Coello Coello, A. Hernandez-Aguirre, 'Extraction and Reuse of Design Patterns from Genetic Algorithms using CaseBased Reasoning', Soft Computing, 9, 44-53, (2005).

[31] J. Prentzas, I. Hatzilygeroudis, 'Categorizing Approaches Combining Rule-Based and Case-Based Reasoning’, Expert Systems, 24, 97-122, (2007).

[32] E.L. Rissland, D.B. Skalak, 'CABARET: Rule Interpretation in a Hybrid Architecture', International Journal of Man-Machine Studies, 34, 839-887, (1991).

[33] D. Rossille, J.-F. Laurent, A. Burgun, 'Modeling a Decision Support System for Oncology using Rule-Based and Case-Based Reasoning Methodologies', International Journal of Medical Informatics, 74, 299-306, (2005).

[34] K.M. Saridakis, A.J. Dentsoras, 'Case-DeSC: A System for CaseBased Design with Soft Computing Techniques’, Expert Systems with Applications, 32, 641-657, (2007).

[35] S.C.K. Shiu, S.K. Pal, 'Case-Based Reasoning: Concepts, Features and Soft Computing’, Applied Intelligence, 21, 233-238, (2004).

[36] I. Watson, 'Case-Based Reasoning is a Methodology not a Technology’, Knowledge-Based Systems, 12, 303-308, (1997)

[37] P. Wriggers, M. Siplivaya, I. Joukova, R. Slivin, 'Intelligent Support of Engineering Analysis using Ontology and Case-Based Reasoning', Engineering Applications of Artificial Intelligence, 20, 709-720, (2007).

[38] H.-L. Yang, C.-S. Wang, 'Two stages of case-based reasoning Integrating genetic algorithm with data mining mechanism', Expert Systems with Applications (to appear).

[39] F.-C. Yuan, C. Chiu, 'A Hierarchical Design of Case-Based Reasoning in the Balanced Scorecard Application', Expert Systems with Applications (to appear). 